\title{
Commonalities and differences in similarity comparisons
}

\author{
ARTHUR B. MARKMAN \\ Columbia University, New York, New York \\ and \\ DEDRE GENTNER \\ Northwestern University, Evanston, Illinois
}

\begin{abstract}
Similarity is a central component of many cognitive processes. Current research suggests that similarity is well characterized as a comparison of structured representations. This process yields commonalities, differences related to the commonalities (alignable differences), and differences not related to the commonalities (nonalignable differences). In the first study, further evidence for this tripartite distinction is provided in a commonality and difference listing study involving pairs of pictures. This study indicates that alignable differences rather than nonalignable differences are central to the comparison process by virtue of their connection to the commonalities. The second study further demonstrates that alignable differences count more against the similarity of a pair than do nonalignable differences. We end by discussing implications of the distinction between alignable and nonalignable differences for other cognitive processes involving comparisons.
\end{abstract}

Similarity comparisons are ubiquitous in cognitive processing. New items are categorized on the basis of their similarity to a stored category representation or to category exemplars (Smith \& Medin, 1981). New problems are solved by using procedures taken from previous problems that were similar (Bassok, 1990; Holyoak \& Koh, 1987; Keane, 1988; Kolodner, 1993; Novick, 1988, 1990; Ross, 1989). Decisions may be based on the similarity of the current situation to the situation that would result from a choice (Smith \& Osherson, 1989). To the degree that we understand how similarity comparisons are carried out, we may also gain insight into other cognitive processes that involve similarity.

Recent research casts similarity as an alignment of structured representations akin to the comparison process involved in analogical reasoning (Falkenhainer, Forbus, \& Gentner; 1989; Gentner \& Markman, 1994, 1995; Goldstone, 1994; Goldstone \& Medin, 1994a; Goldstone, Medin, \& Gentner, 1991; Markman \& Gentner, 1993a, 1993b; Medin, Goldstone, \& Gentner, 1993). This work extends the long-held assumption that the similarity of a pair of items increases with its commonalities and decreases

Order of authorship was determined by chance. This work was supported by National Science Foundation Grant BNS-909-6259, awarded to D.G. and Doug Medin, and by Office of Naval Research Grant N00014-89-J1272, awarded to D.G. and Ken Forbus. We thank Ken Forbus, Doug Medin, Brian Ross, Tom Ward, Ed Wisniewski, two anonymous reviewers, and the Similarity and Analogy Group at Northwestern for helpful discussions during the evolution of this project. We also thank Linda May for transcribing the data from Experiment 1. Correspondence should be addressed to A. B. Markman, Department of Psychology, 406 Schermerhorn Hall, Columbia University, New York, NY 10027 (e-mail: markman@psych.columbia.edu) with its differences (e.g., Tversky, 1977) by distinguishing between two types of differences: those connected to the commonalities (called alignable differences) and those not connected to the commonalities (called nonalignable differences). The studies in this paper broaden our knowledge about these two types of differences with evidence from studies involving picture pairs. The first study replicates earlier work done with word pairs. The second study focuses on the relative impact of alignable and nonalignable differences on rated similarity. To place these studies in perspective, we outline the view of the comparison process that has emerged from recent research and use it to derive a set of four predictions to be tested.

\section{A Structural Alignment View of Similarity}

Recent research on the structural alignment process makes assumptions both about the nature of cognitive representations and about the processes that compare those representations. On the representation side, this view assumes that representations are composed of object descriptors (called attributes), objects (or entities), functions, and relations between representational elements. ${ }^{1}$ For example, the simple geometric configurations in Figure $1 \mathrm{~A}$ can be characterized by the structured representations graphed in Figure $1 \mathrm{~B}$. Here, the circle and square (entities) are described by such attributes as shaded and striped. These entities form the arguments of the relation above (circle, square). A key aspect of this representation is that connections between elements in the representation are encoded explicitly with labeled relations.

On the processing side, it is assumed that similarity comparisons involve a mechanism similar to the structuremapping process proposed for analogy (Falkenhainer et al., 1989; Gentner, 1983, 1989; Hofstadter \& Mitchell, 


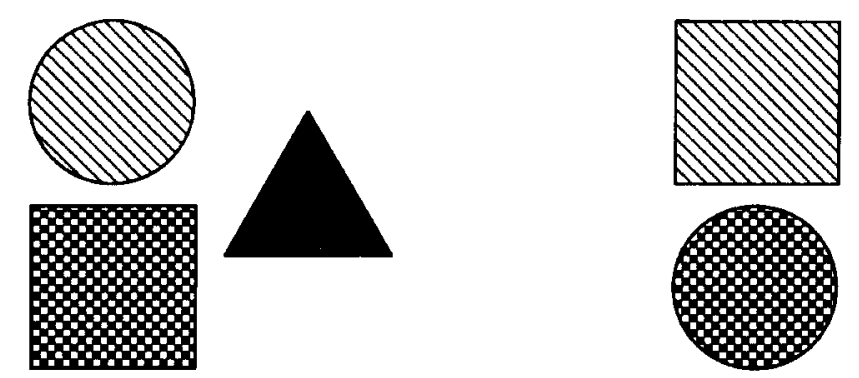

$\mathbf{A}$

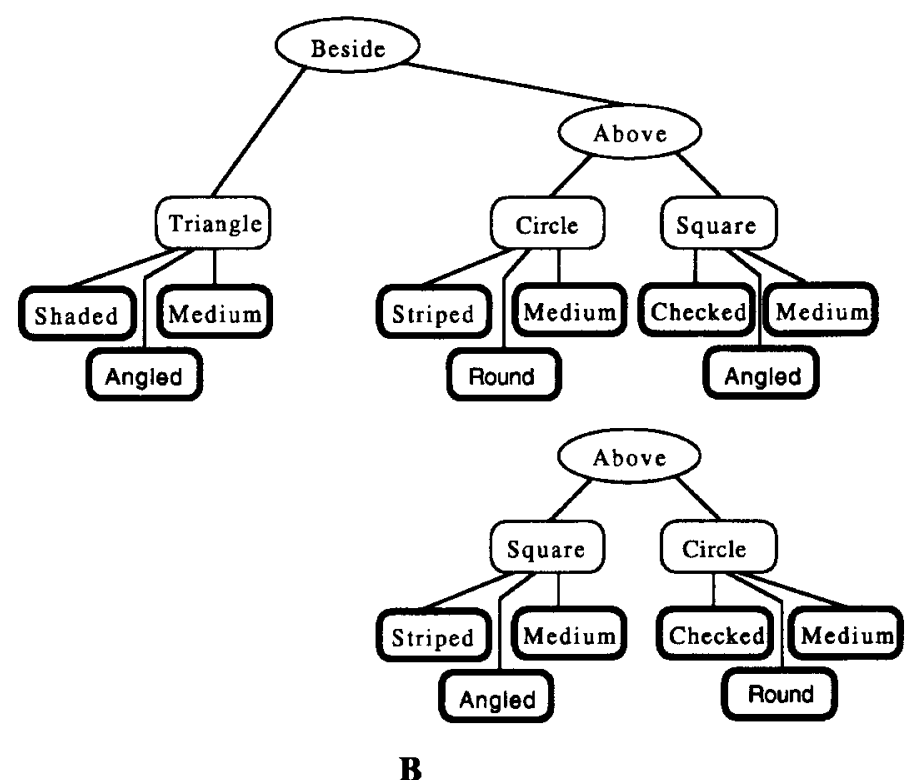

Figure 1. (A) Simple configurations of objects and (B) simple relational structure describing the configurations.

1994; Holyoak \& Thagard, 1989; Keane, 1988; Medin et al., 1993). Intuitively, the main idea is that the comparison of complex structures involves not only the matching of features, but also the determination of which features and objects play the same role in the relational system. Specifically, the structural alignment process seeks maximal structurally consistent matches, that is, those that satisfy the constraints of parallel connectivity and one-to-one mapping. Parallel connectivity requires that matching relations have matching arguments. For example, if the above $(x, y)$ relations in Figure $1 \mathrm{~B}$ are placed in correspondence, then the circle in the top representation and the square in the bottom representation must also be placed in correspondence because both are the first arguments to the relation (i.e., they are on top). Similarly, the square and the circle in the top and bottom representations, respectively, must be placed in correspondence because they are the second arguments of the relation (i.e., they are on the bottom). One-to-one mapping states that each element in one representation can be matched to, at most, one element in the other representation. For example, if the circle in the top representation in Figure $1 \mathrm{~B}$ is mapped to the square in the bottom representa- tion (because both are on top), then it cannot simultaneously be matched to the circle in the bottom representation.

In previous work, we established that the alignment process yields commonalities and two kinds of differences: those differences that are linked to the commonalities (called alignable differences) and those differences that are unrelated to the commonalities (called nonalignable differences) (Gentner \& Markman, 1994; Markman \& Gentner, 1993a). For example, if the two drawings in Figure $1 \mathrm{~A}$ are matched on the basis of their common above $(x, y)$ relation (which would then be a commonality of the pair), then the fact that there is a circle on the top in one case and a square on the top in the other would be considered an alignable difference. Subjects typically state such alignable differences as different values on a common predication: for example, "There is a circle on top in one and a square on top in the other." In contrast, the triangle in the left drawing, which does not correspond to anything in the right drawing, is a nonalignable difference. It is not part of the alignable structure, as shown in Figure 1B. Subjects typically state that these differences are aspects of one item that are not present in the other: for example, "There is a triangle in 
one configuration but not in the other." More fundamentally, alignable differences are corresponding elements that are unlike, whereas nonalignable differences are noncorresponding elements. On this view, the classification of differences as alignable or nonalignable is done relative to the way in which the two situations are aligned: that is, relative to the perceived common structure.

This tripartite output of the comparison process departs from the predictions of feature-based models of comparison (e.g., Tversky, 1977), which distinguish only between commonalities (matching features) and differences (mismatching features). In addition, most feature-matching models assume that the commonalities and differences of a pair form independent sets. In contrast, on the structural alignment view, alignable differences cannot be determined until the commonalities of the pair are known. Thus, to the degree that the distinction between alignable differences and nonalignable differences proves important, representational and processing assumptions beyond those posited by simple featural models are required.

The structural alignment view makes four key claims concerning commonalities and differences in similarity. The first two predictions derive from the connection between commonalities and alignable differences. First, there should be a numerical relationship between commonalities and alignable differences: pairs with many commonalities should have many alignable differences, and pairs with few commonalities should have few alignable differences. Second, because alignable differences are mismatching representational elements that are connected to the system of matching elements, alignable differences should be conceptually linked to the commonalities from which they are determined.

The third and fourth predictions concern the distinction between alignable differences and nonalignable differences. For the third, subjects should focus on alignable differences rather than on nonalignable differences in comparisons. For example, they should find it easier to list alignable differences than to list nonalignable differences. This last prediction follows from the assumption that similarity comparisons maximize the commonalities of a pair (Krumhansl, 1978; Sjöberg, 1972), combined with our claim that alignable differences are more closely connected to commonalities than are nonalignable differences. For the fourth, by the same reasoning, alignable differences should also be more important in evaluating similarity comparisons than should nonalignable differences. That is, all else being equal, alignable differences should count more against the similarity of a pair than should nonalignable differences. At first, this prediction seems to lead to a paradox. The pairs that have the most commonalities (and hence are most similar) are also the pairs with the most alignable differences (and hence have the most salient differences). However, it is well established that commonalities are more important to the perception of similarity than are differences (Tversky, 1977). Thus, the amount that commonalities increase rated similarity is greater than the amount that differences decrease similarity, so that pairs with many commonalities and alignable differences are perceived as being more similar than pairs with few commonalities and few alignable differences.

We have obtained evidence for the first three of these predictions, but not for the fourth. In one study, we asked subjects to list commonalities and differences for word pairs at different levels of similarity (Markman \& Gentner, 1993a). In support of the first claim, the subjects listed many alignable differences (along with many commonalities) for similar pairs and few alignable differences (and few commonalities) for dissimilar pairs. This finding was reflected in a positive correlation between the number of listed alignable differences and the number of listed commonalities.

Consistent with the second prediction, there were conceptual relationships between commonalities and alignable differences. For example, subjects listing commonalities for the pair car/motorcycle often said, "Both have wheels," while subjects listing differences for the same pair often listed the alignable difference, "Cars have four wheels and motorcycles have two wheels." A sorting task was used to confirm this relationship. The subjects were shown the commonalities and differences listed for a pair and asked to put together any that they felt were conceptually related. The subjects sorted alignable differences (but not nonalignable differences) with the commonalities (Markman \& Gentner, 1993a). As predicted, no relationship between commonalities and nonalignable differences was found by any of these criteria. The subjects listed fewer nonalignable differences for similar pairs than for dissimilar pairs, there was no significant correlation between the number of listed commonalities and the number of nonalignable differences, and commonalities and nonalignable differences were rarely conceptually linked.

The third claim - that comparisons make alignable, but not nonalignable, differences salient - leads to the counterintuitive prediction that people should find it easier to list differences for high-similarity pairs than for low-similarity pairs. We tested this by giving subjects a large number of word pairs - half of high similarity and half of low similarity - and asking them to list one difference for as many pairs as possible in 5 min (Gentner \& Markman, 1994). As predicted, the subjects in this "speeded difference task" chose to list differences for many more high-similarity pairs than they did for low-similarity pairs $(M=11.4$ and $M=$ 5.9 , respectively). Furthermore, this effect was concentrated in the alignable differences. These were listed about three times as often as nonalignable differences overall and accounted for the superiority of the high-similarity pairs. The pattern that subjects list more alignable differences than nonalignable differences has held across all our commonality and difference listing studies (Gentner \& Markman, 1994; Markman \& Gentner, 1993a). This pattern, together with the striking finding that subjects in the speeded differences task preferred to list differences for high-similarity pairs, supports the claim that subjects find alignable differences more natural than nonalignable differences.

However, the fourth prediction-that alignable differences should decrease similarity more than nonalignable differences - remains unsupported. In our previous stud- 
ies, regression analyses showed that rated similarity increased with commonalities and decreased with differences, consistent with Tversky's (1977) findings. Also consistent with Tversky's findings, the positive contribution of commonalities was larger than the negative contribution of differences. However, when we separated differences into alignable and nonalignable differences, we found that (1) the coefficient for alignable differences was not significantly different from zero, and (2) the regression coefficient with similarity was significantly negative for nonalignable differences. These findings are the opposite of the predicted pattern that alignable differences should count more against similarity than should nonalignable differences.

These two discrepant findings may be explained by two problems with the use of regression analysis to examine how commonalities and differences are combined to form an impression of similarity. First, in every study we have done, we have obtained a significant positive correlation between the number of listed commonalities and the number of listed alignable differences. Therefore, a linear model cannot separate the (positive) impact of commonalities on similarity from the (predicted negative) impact of alignable differences on similarity. In part, this explanation may also account for why the coefficient for nonalignable differences is larger than the one for alignable differences. The second potential problem is the well-known conundrum that correlational analyses establish neither a causal relationship nor the direction of causality should a causal relationship exist. Thus, the fact that the regression coefficients for nonalignable differences are larger than we might have expected in advance may reflect a reverse causal link between similarity and nonalignable differences. Subjects may be most likely to list nonalignable differences for low-similarity comparisons, for which they can think of nothing better to do. On this view, the regression analysis reflects a relationship between the similarity of a pair and the number of nonalignable differences that are listed, but not the weight given to those nonalignable differences in similarity comparisons.

Clearly, our earlier studies leave us with a puzzle. The structure-mapping approach successfully predicts that (1) there is a positive relationship between the number of listed commonalities and alignable differences for a given pair, (2) alignable differences are conceptually related to the commonalities from which they were derived, (3a) alignable differences are listed more naturally than are nonalignable differences, and (3b) differences are listed more easily for similar pairs than for dissimilar pairs. However, if the structural alignment view is correct, then alignable differences should count more against similarity than should nonalignable differences. How can this issue be resolved?

To approach this question, we decided to repeat the commonality and difference listing studies with picture pairs, in which the entire set of differences is visually available to subjects, making it easy to list nonalignable as well as alignable differences. Thus, if the negative correlation between similarity and nonalignable differences found in our previous studies was caused by neglect of nonalignable differences, then it should no longer hold with these pic- torial stimuli. The other predictions derive from the previous discussion. If the principles of structure-mapping operate here as with the verbal materials we have used earlier, then we should find positive correlations between commonalities and alignable differences and between similarity and commonalities. Thus, there should be a positive correlation between similarity and alignable differences.

The use of picture pairs also allows us to escape some limitations of the word-pair materials used in previous studies. With word pairs, the conceptual representations must be inferred from subjects' feature listings. Because subjects typically do not list relations between attributes, the prediction that alignable differences are connected to structural commonalities requires additional analyses (like the sorting task described above; Markman \& Gentner, 1993a). By using picture pairs, we can manipulate the individual components and their interrelations within the scenes. A related advantage is that picture pairs permit us to vary whether a difference is alignable or nonalignable, and thus lend themselves to separating the effects of alignable and nonalignable differences on subjects' judgments of similarity. Finally, with pairs of concepts, there is variability due to variations in subjects' conceptual representations of these words as a function of past experience. Since much of the information relevant to the picture pairs is derived directly from the pictures themselves, there should be less variability between subjects than in previous studies.

Thus, in Experiment 1, we investigated whether picture pairs would yield the same pattern of commonality and difference listings already obtained with word pairs in support of the first three predictions of structural alignment. In this study, subjects listed commonalities and differences of pictorial scenes rather than of individual concepts. If these scenes yielded the same patterns across commonalities and differences as do word pairs, we would be able to generalize our results beyond preexisting taxonomic category representations. Furthermore, if the results of this first study showed the same effects as the previous studies, then the stage would be set for a systematic manipulation of alignable and nonalignable differences to test the fourth prediction-that alignable differences count more than nonalignable differences in judgments of similarity.

A subsidiary goal of this study was to examine factors that promoted correspondences between items in each picture. To this end, we created eight triads of pictures like the one in Figure 2. From these triads, two sets of picture pairs were created. The picture in A was paired with the picture in $B$ in one stimulus set and with the picture in $C$ in the other stimulus set. This design allowed us to compare how the same item in one picture would be matched to different items in different comparisons. We also wanted to have pairs that spanned a range of similarities, so the stimulus set included four low-similarity pairs (formed by arbitrarily pairing the eight pictures from the triads that were not already used) and four high-similarity pairs in which similar objects were placed in a similar relational structure (i.e., literally similar pairs; Gentner, 1983, 1989).

In Experiment 1, we asked whether the pictorial stimuli would support the first three predictions of structural 


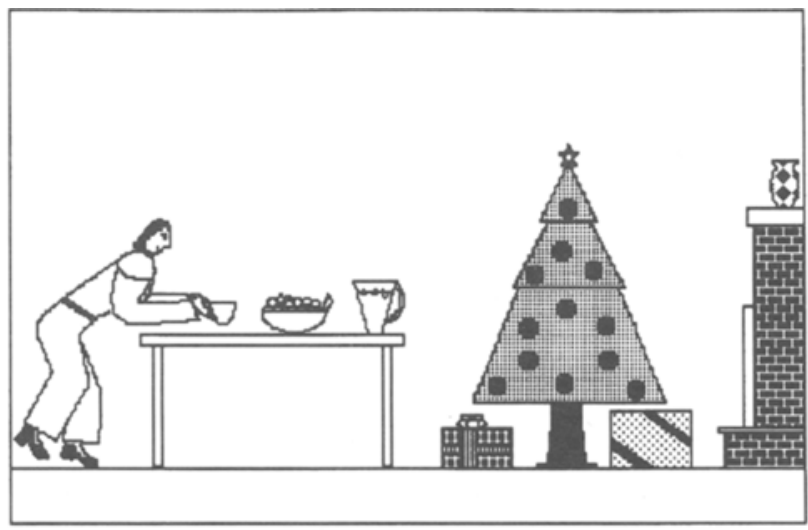

A

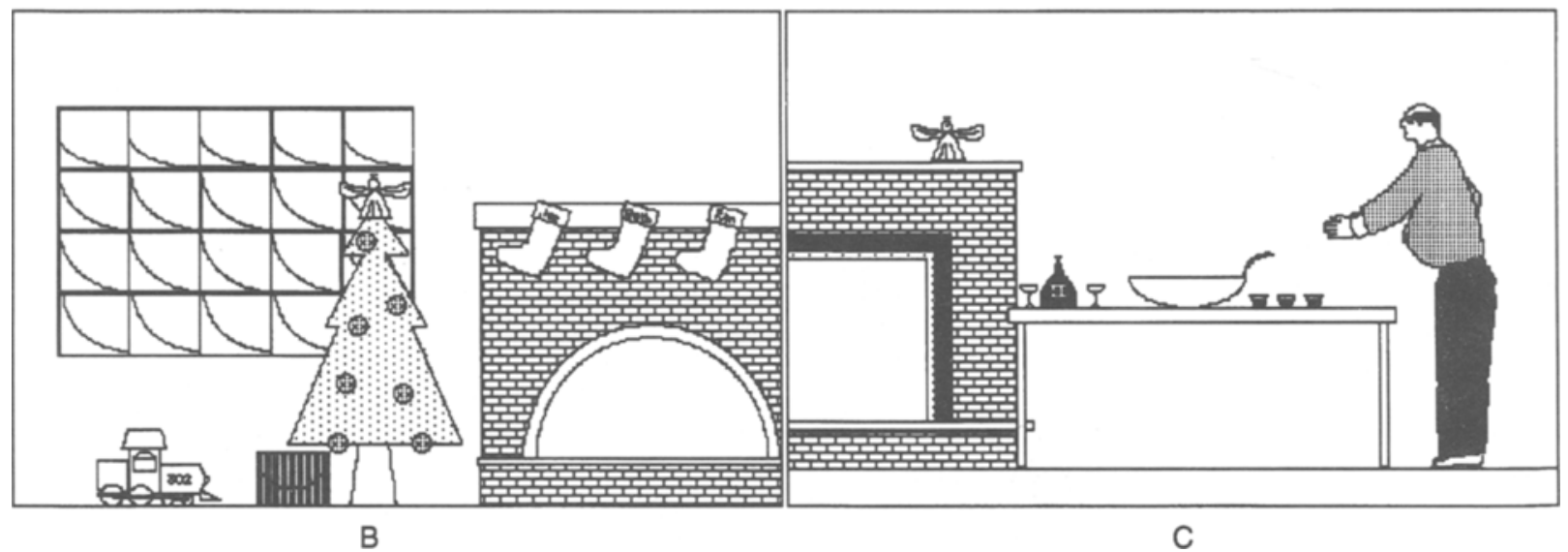

Figure 2. Sample triad of pictures used in Experiment 1.

alignment. First, as in previous studies, the number of alignable differences listed for a pair should be positively related to the number of commonalities listed (and thus both should show a positive relationship to rated similarity). Second, the alignable differences should be conceptually related to the way that elements of the pictures were placed in correspondence. Third, comparisons should focus on alignable rather than nonalignable differences. If these patterns held for pictorial pairs as they do for word pairs, this would strengthen the claim that commonalities, alignable differences, and nonalignable differences were the result of a general similarity-comparison process.

\section{EXPERIMENT 1}

\section{Method}

Subjects. The subjects in this study were 40 undergraduates from the University of Illinois who were given course credit for their participation.

Materials. Two between-group stimulus sets were created, each containing 16 pairs of pictures. The heart of each stimulus set was a group of 8 triads of pictures (like the one in Figure 2). In the first stimulus set, the target picture (e.g., Figure $2 \mathrm{~A}$ ) was paired with one of the other two pictures (e.g., Figure 2B), and in the second set, the target picture was paired with the other picture (e.g., Figure $2 \mathrm{C}$ ). The eight remaining pictures were arbitrarily paired to form four more pairs. Finally, four pairs in which similar objects participated in sim- ilar causal relations (literally similar pairs) were included in each set to ensure that the sets covered a wide range of similarities. All pictures were drawn with a computer drawing package and were mounted individually on colored paper.

Procedure. The subjects were run individually at a table in a small room. At the start of a trial, the experimenter placed a pair of pictures in front of the subject and asked him/her to list either the commonalities or the differences of the pair. The subjects listed either the commonalities or the differences of a pair, but not both. Thus, 2 subjects were required to get a complete set of commonality and difference listings for each stimulus set.

The subjects listed the commonalities and differences aloud into an audio tape for $1 \mathrm{~min}$. The experimenter timed each trial with a watch. At the end of $1 \mathrm{~min}$, the experimenter signaled the subject to stop, and placed the next pair of pictures in front of him/her. Generally, the subjects finished listing commonalities and differences well before the minute was up. A short break was given after eight trials. After listing commonalities or differences for all 16 pairs, the subject saw each pair a second time and rated its similarity on a 9-point scale. ${ }^{2}$ The session took approximately $25 \mathrm{~min}$ to complete.

Design. Similarity of the picture pairs was manipulated within subjects, so that each subject saw pairs that spanned a range of similarities. For purposes of the analyses, the stimuli were divided into three groups (i.e., low similarity, medium similarity, and high similarity) on the basis of subjects' similarity ratings. There were 2 between-subject stimulus sets and 10 random stimulus presentation orders, with 4 subjects seeing each order. The dependent measures were the number of commonalities, alignable differences, and nonalignable differences listed by the subjects, as well as the rated similarity of the items. 
Scoring. The protocols were transcribed and scored using the procedure we had employed previously (Markman \& Gentner, 1993a). For commonality listings, one commonality was counted for each item subjects listed as part of both pictures: for example, "Both have Christmas trees." Difference listings were separated into alignable differences and nonalignable differences. An item was counted as an alignable difference if subjects mentioned that the pictures differed along some common aspect: for example, "One picture has a vase and the other has an angel." An item was scored as a nonalignable difference if the subject mentioned an aspect of one of the pictures and simply negated it for the other picture (or failed to mention the other picture at all): for example, "One picture has a train [and the other does not]." As discussed in our previous work (Markman \& Gentner, 1993a), this scoring procedure is conservative in that subjects could see a feature as an alignable difference but fail to place it in the appropriate linguistic form. However, this conservatism should only make our hypotheses more difficult to support by uniformly decreasing the level of alignable differences (which are assumed to be the more important kind of difference). One rater scored the entire data set and a second naive rater scored a random $20 \%$ subsample of the data. There was $94 \%$ agreement between the raters.

\section{Results}

Prediction 1: Numerical relationships between commonalities and differences. We begin with an examination of the numerical relationships between listed properties. Table 1 shows the mean number of listed commonalities, alignable differences, and nonalignable differences for pairs of low, medium, and high similarity (as defined by subjects' ratings). The low-similarity pairs had a mean rated similarity of 1.94, the medium-similarity pairs had a mean rated similarity of 4.79 , and the high-similarity pairs had a mean rated similarity of 7.34 . Furthermore, as planned, the two stimulus sets each spanned a range of similarities. For Set 1, the rated similarities ranged from a low of 1.17 to a high of 7.98 with a mean of 4.13 . For Set 2 , the range was 1.46 to 8.00 with a mean of 5.10 .

For these results to be compatible with those of previous studies, high-similarity pairs should have more listed commonalities than low-similarity pairs. Indeed, as the similarity of the pairs increased, the number of listed commonalities increased $[F(2,29)=51.02, p<.001]$, with significantly more commonalities listed for high-similarity pairs $(M=6.53)$ than for medium- $(M=4.24)$ or lowsimilarity pairs $(M=1.54)$ and more commonalities listed for medium-similarity pairs than for low-similarity pairs (all $p s<.05$, Bonferroni). Similarly, the number of alignable differences listed increased with similarity $[F(2,29)=$ $6.87, p<.05]$. More alignable differences were listed for high-similarity $(M=3.20)$ and medium-similarity $(M=$ 2.97) pairs (which did not differ from one another) than for low-similarity pairs $(M=1.91)$ (both $p$ s $<.05$, Bonferroni). In contrast, the number of listed nonalignable differences did not differ significantly across the three levels of similarity $[F(2,29)=2.28, p>.10]$.

This pattern is reflected in correlational analyses performed on the mean number of listed items and mean similarity ratings of the pairs in each set. As predicted, there was a significant positive correlation between rated similarity and the number of commonalities listed for each pair $[r(14)=0.98$ for Stimulus Set 1 and $r(14)=0.91$ for Stimulus Set 2, both $p s<.001]$. Furthermore, as expected, there was a significant positive correlation between rated similarity and the number of alignable differences listed $[r(14)=0.71$ for Stimulus Set $1, r(14)=0.50$ for Stimulus Set 2 , both $p$ s $<.05]$. In contrast, no significant correlation was obtained between rated similarity and the number of nonalignable differences listed $[r(14)=-0.07$ for Stimulus Set 1 and $r(14)=0.12$ for Stimulus Set 2, both $p s>.10]$.

Correlational analyses can also be used to examine the claim that pairs with many commonalities have many alignable differences, while pairs with few commonalities have few alignable differences. Consistent with this prediction, there was a positive correlation between the number of listed commonalities and the number of listed alignable differences $[r(14)=0.69$ for Stimulus Set $1, r(14)=$ 0.58 for Stimulus Set 2 , both $p$ s $<.05$ ]. In contrast, no significant correlation was obtained between commonalities and nonalignable differences $[r(14)=-0.06$ for Stimulus Set $1, r(14)=0.03$ for Stimulus Set 2, both $p s>.10$ ]. As in previous findings, this pattern suggests that the alignable differences are related to the commonalities but that the nonalignable differences are not. ${ }^{3}$

Prediction 2: Conceptual relationship between commonalities and differences. The defining characteristic of alignable differences is that they are connected to commonalities. Consistent with the first prediction of structural alignment, we have already demonstrated that alignable differences are numerically related to commonalities. We now turn to the second prediction with an examination of conceptual relationships between alignable differences and the correspondences from which they are derived. We examined all of the consensus differences, defined as those differences listed by at least 5 subjects. The differences were compared with the original pictures to determine what type

Table 1

Mean Commonalities, Alignable Differences, and Nonalignable Differences as a Function of Pair Similarity in Experiment 1

\begin{tabular}{lcccccc}
\hline $\begin{array}{c}\text { Pair } \\
\text { Similarity }\end{array}$ & $\begin{array}{c}\text { Number of } \\
\text { Pairs }\end{array}$ & $\begin{array}{c}\text { Rated } \\
\text { Similarity }\end{array}$ & Commonalities & $\begin{array}{c}\text { Alignable } \\
\text { Differences }\end{array}$ & $\begin{array}{c}\text { Nonalignable } \\
\text { Differences }\end{array}$ & $\begin{array}{c}\text { Total } \\
\text { Differences }\end{array}$ \\
\hline Low & 10 & 1.94 & 1.54 & 1.91 & 2.99 & 4.90 \\
Medium & 13 & 4.79 & 4.24 & 2.97 & 2.23 & 5.20 \\
High & 9 & 7.34 & 6.53 & 3.20 & 3.36 & 6.56 \\
\hline
\end{tabular}

Note Low-similarity pairs had a mean similarity rating less than 3.66, medium-similarity pairs had a mean similarity rating between 3.67 and 6.33 , and high-similarity pairs had a mean similarity rating greater than 6.34 
of correspondence (if any) was required to determine this difference. For example, in the comparison between Figures $2 \mathrm{~A}$ and $2 \mathrm{~B}$, noticing that both pictures have Christmas trees may lead to the discovery that the ornaments on the trees differ.

In all, there were 72 consensus alignable differences. All of these differences were clearly the result of a particular correspondence between a pair of pictures. Of the 72 consensus alignable differences, $43(60 \%)$ were derived from objects that seem to have been placed in correspondence on the basis of their attribute similarity. For example, all 10 subjects listing differences for the pair made up of Figures $2 \mathrm{~A}$ and $2 \mathrm{~B}$ pointed out that the Christmas trees (which are placed in correspondence on the basis of their perceptual similarity) have different ornaments. The remaining $29(40 \%)$ consensus alignable differences were associated with relational commonalities. For example, $7 / 10$ of the subjects listing differences for the pair made up of Figures $2 \mathrm{~A}$ and $2 \mathrm{C}$ pointed out that there was a vase on top of the fireplace in one picture and an angel on top of the fireplace in the other. Similarly, $7 / 10$ subjects listing differences for the pair made up of Figures $2 A$ and $2 B$ said that the star on top of the tree and the angel on top of the tree were a difference between the pictures. In these two examples, the items placed in correspondence were not perceptually similar, but rather played like roles in a matching relation between the pictures, evincing the hallmark of analogical comparisons.

In contrast to the alignable differences, the nonalignable differences were generally not related to the correspondence between the pictures. In all, there were 33 consensus nonalignable differences. Of these, $27(82 \%)$ were not obviously related to the commonalities of the scenes. For example, $7 / 10$ subjects listing differences for the pair in Figures 2A and 2B pointed out that there was a window in one picture, but not in the other. Clearly, the window is a salient perceptual aspect of the picture, but it does not have a correspondence with any of the elements in the other picture. The remaining $6(18 \%)$ were situations in which two items were placed in correspondence, leading to the perception of a missing property for one of the items. For example, $6 / 10$ subjects listing differences for the pair in Figures 2A and 2B pointed out that there were stockings in one picture but not in the other. This difference may have been salient because there were corresponding fireplaces in each scene, only one of which had stockings hanging from it. It is an open question as to whether there is a psychological difference between these two subtypes of nonalignable differences (see also Markman \& Medin, 1995, for a discussion of this issue).

Prediction 3: Alignable differences favored over nonalignable differences. This study has provided support for the first two predictions of structural alignment. However, there is an interesting difference between the results of this study and those of the previous studies with word pairs (Gentner \& Markman, 1994; Markman \& Gentner, 1993a). In our previous work, there were generally fewer nonalignable differences listed than alignable differences. In contrast, in the current study, using pictures, the over- all number of nonalignable differences listed $(M=2.79)$ was roughly the same as the number of alignable differences listed $(M=2.70)$. This finding does not seem compatible with the claim that comparisons focus on alignable differences rather than on nonalignable differences. In casting about for the reason for this difference, we considered two ways that the picture pairs differed from the word pairs. First, in the word studies, the words denoted individual concepts, while the pictures used here depicted relatively complex scenes. Second, for the word pairs, the representations of both items had to be kept in working memory, whereas for the current picture pairs, the pairs remained in front of the subjects while they were listing differences. Thus, subjects in the word task may have been limited to considering material in a narrower attentional focus than were subjects in our current picture task.

This reasoning suggests a further analysis. We have argued that comparisons generally focus on those commonalities and alignable differences connected to the most systematic matching structure (cf. Gentner, 1989). Alignable differences arise from a set constrained by the matching structure, but nonalignable differences can be drawn from a potentially indefinite set. By this reasoning, we would expect greater between-subject consistency in listing alignable differences than in listing nonalignable differences. One piece of supporting evidence for this claim is that, of the 105 consensus differences described above (i.e., those listed by at least 5 subjects), $72(69 \%)$ were alignable differences and only $33(31 \%)$ were nonalignable differences. This finding is consistent with the claim that comparisons focus subjects on alignable differences, with other differences being optional.

\section{Discussion}

The results of Experiment 1 bear out the predictions of structural alignment. In support of the first prediction, pairs with many commonalities also had many alignable differences, while pairs with few commonalities had few alignable differences. In contrast, there was no significant relationship between the number of commonalities and the number of nonalignable differences listed for a pair. In support of the second prediction, consensus alignable differences were clearly derived conceptually from correspondences between the pictures, while only a small subset of the nonalignable differences appeared to be at all related to the correspondences. This finding reinforces the idea that structural alignment rather than mere feature overlap determines the commonalities and differences of a pair. Finally, in support of the third prediction, there was greater consistency in the listings of alignable differences than in the listings of nonalignable differences, suggesting that the alignable differences were the result of a search for a common relational system, while the nonalignable differences were not.

Commonalities, differences, and similarity: Two pilot studies. These data clearly demonstrate that commonalities and alignable differences are related, but commonalities and nonalignable differences are not. They also provide some evidence that comparisons focus on align- 
able differences rather than on nonalignable differences. However, this study does not permit us to examine the relationship between commonalities, alignable differences, nonalignable differences, and the perception of similarity. As discussed above, regression analyses cannot effectively discriminate between the impact of commonalities on similarity and the impact of alignable differences on similarity. Thus, another methodology is required to address this issue.

The most straightforward test of this hypothesis is a forced-choice task that pits an item serving as an alignable difference against the same item serving as a nonalignable difference. Such a task is illustrated in Figure 3. Here the choice alternatives are nearly identical to the standard, except that a new item (the bird) has been added. In one case, the item is added as a nonalignable difference (here, behind the target), but in the other it is added as an alignable difference (here being shot at by the man). If subjects find that the alignable difference decreases similarity more than does the nonalignable difference, then, if they are asked to select the figure most similar to the standard, they should select the nonalignable difference choice. We conducted a pilot study with 24 subjects, who were given choices involving eight triads structured like the one in Figure 3. Con- sistent with the idea that alignable differences decrease similarity more than do nonalignable differences, on $88 \%$ of the trials (significantly above chance), subjects selected the nonalignable difference choice as most similar to the standard. Further support for this focus on the alignable difference was that $14 / 24$ subjects justified their responses after the conclusion of the experiment with such statements as, "The object of the action was important; anything else was unnecessary" and "[I looked to see that ] the object that was being acted on stayed the same."

One problem with this methodology is that the alignable difference replaces the object in the standard. For example, in the alignable difference choice in Figure 3, the bird replaces the target. Thus, subjects could be selecting the picture that contains all of the same elements as the standard. This objection can be countered by using triads like the one in Figure 4. Here, the object replaced by the alignable difference still appears in the picture in a different location (here the target now hangs on the tree). In a second pilot study, 16 subjects were each presented with eight triads like the one in Figure 4. As expected, the subjects selected the nonalignable difference choice as most similar on $95 \%$ of trials (again more than would be ex-

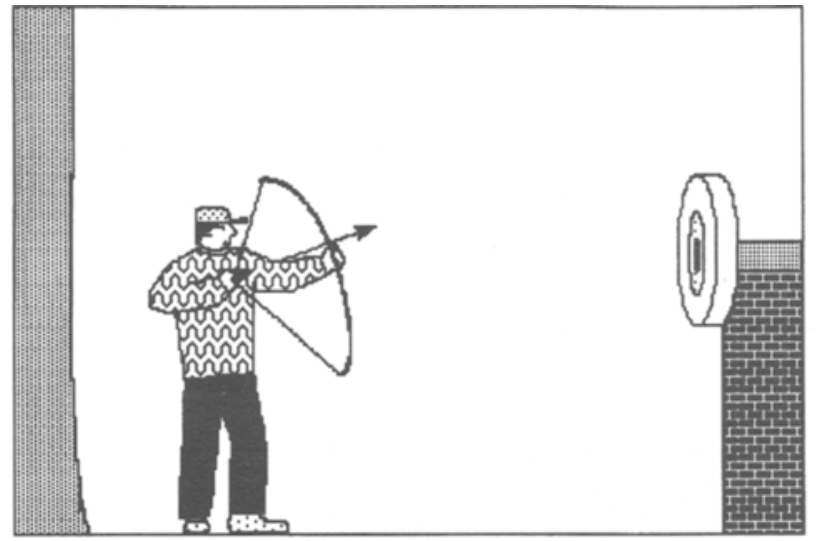

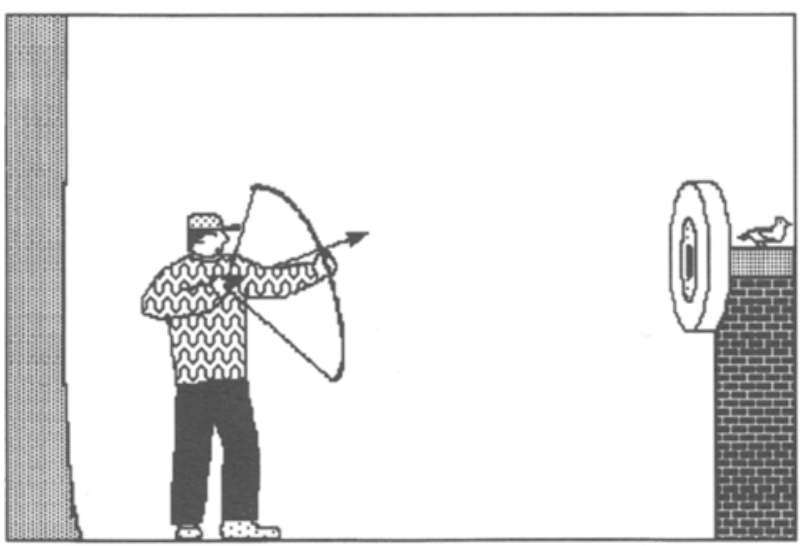

Nonalignable difference choice

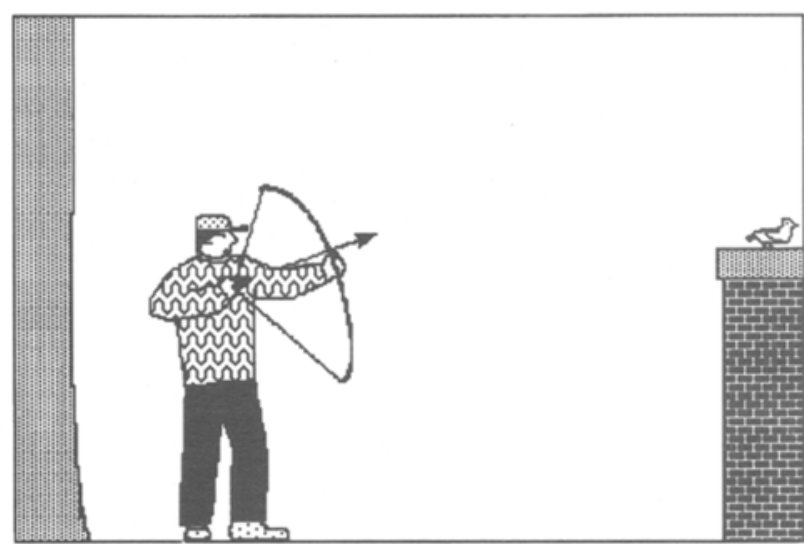

Alignable difference choice

Figure 3. Sample set of pictures that could be used to determine whether alignable differences or nonalignable differences count more against rated similarity. 


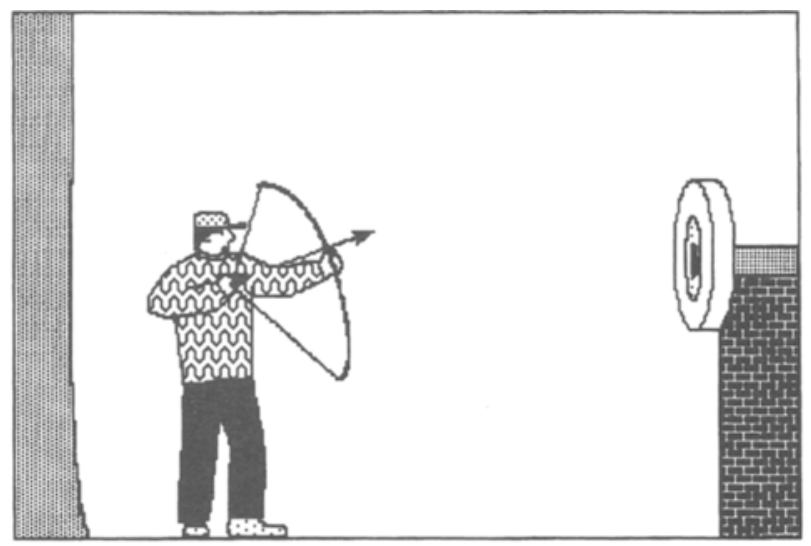

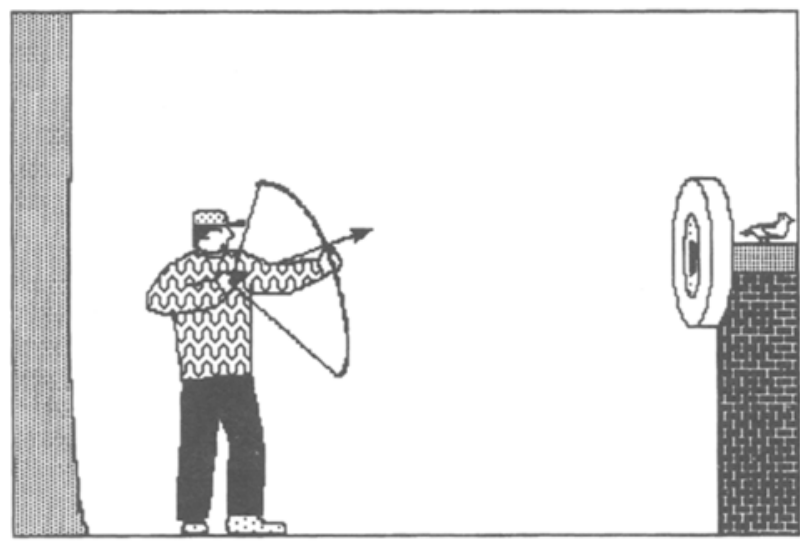

Nonalignable difference choice

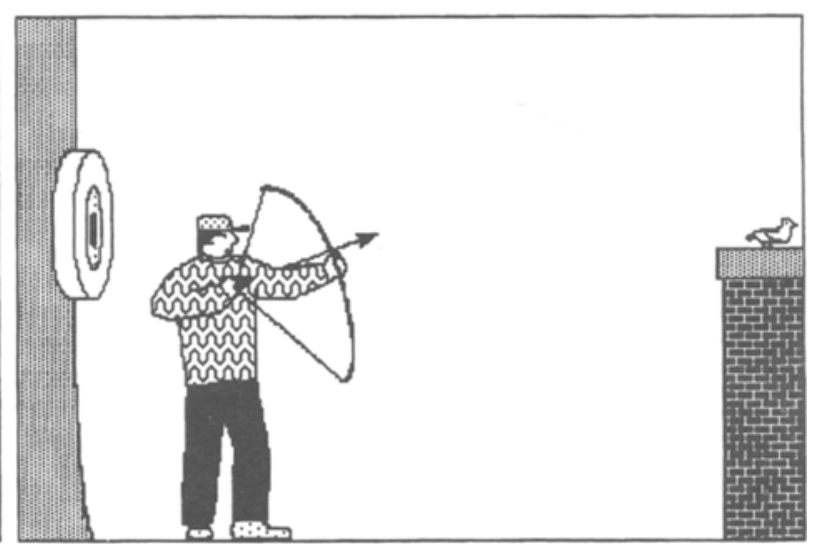

Alignable difference choice

Figure 4. Another set of pictures that could be used to assess the relative impact of alignable and nonalignable differences on similarity. Here, the target in the alignable difference choice is located on the tree.

pected by chance). Once again, however, there is a second explanation. In this study, subjects may be selecting the item that has all of the elements of the original study in their original location. Clearly these pilot studies are consistent with the idea that alignable differences decrease similarity more than do nonalignable differences. Unfortunately, it is not possible to rule out all alternative explanations for the data in a single forced-choice study.

Because of the difficulty in equating the comparison figures on all dimensions except the item appearing as an alignable difference or as a nonalignable difference, we adopted a different methodology. Understanding the logic of this method requires a deeper examination of the prediction that alignable differences count more against similarity than do nonalignable differences. One way to interpret this claim is that any alignable difference counts more against similarity than does any nonalignable difference. However, the prediction of structural alignment is more subtle. The impact of a given difference is greater if it is connected to the common structure than if it is not, because subjects focus more attention on alignable differences than on nonalignable differences when making comparisons. If the corresponding objects that make up an alignable difference are similar (within the context of the relational role they fill), then the alignable difference should not decrease similarity much. If the corresponding objects are dissimilar, then the alignable difference should decrease similarity greatly. In contrast, because nonalignable differences have no corresponding objects in the other scene, there should be considerably less variation in similarity as the object changes. ${ }^{4}$

Thus, we suggest that variations in an alignable difference will affect similarity more than variations in a nonalignable difference. We can test this proposal empirically using a set of pictures like the one in Figure $5 .^{5}$ The standard (Figure 5A) depicts a robot fixing a car. This standard is compared with a picture that contains a particular alignable difference (e.g., a robot fixing something else) or to one that contains a nonalignable difference (e.g., some other item on the floor near the robot). An item added as an alignable difference can be either similar (as in Figure $5 \mathrm{~B}$, in which the robot now fixes a truck) or dissimilar (as in Figure 5C, in which the robot fixes another robot). The same two items (a truck or a robot) can be added as a nonalignable difference as well (as in Figures $5 \mathrm{D}$ and $5 \mathrm{E}$, respectively). The central prediction of structural alignment is that the variation of the item will matter much more for alignable differences than for non- 


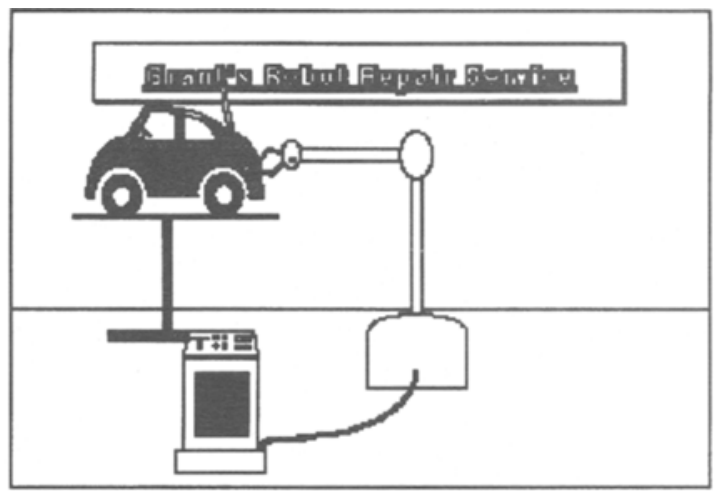

(A)

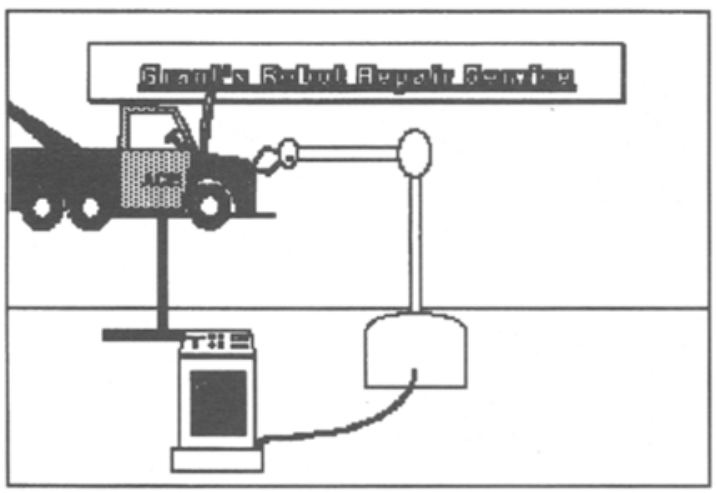

(B)

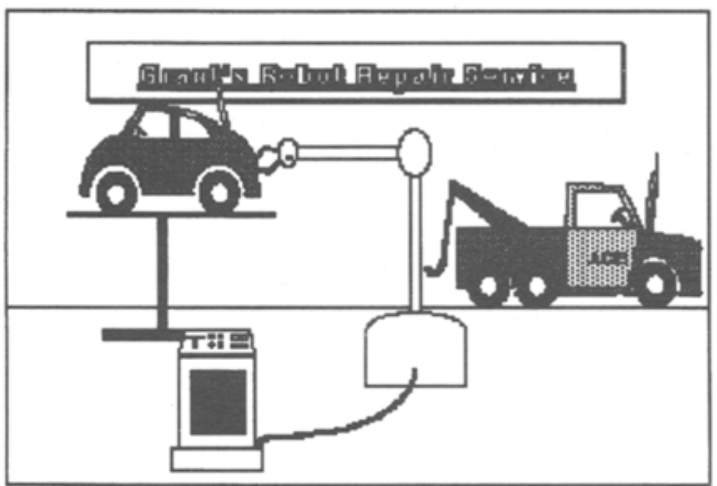

(D)

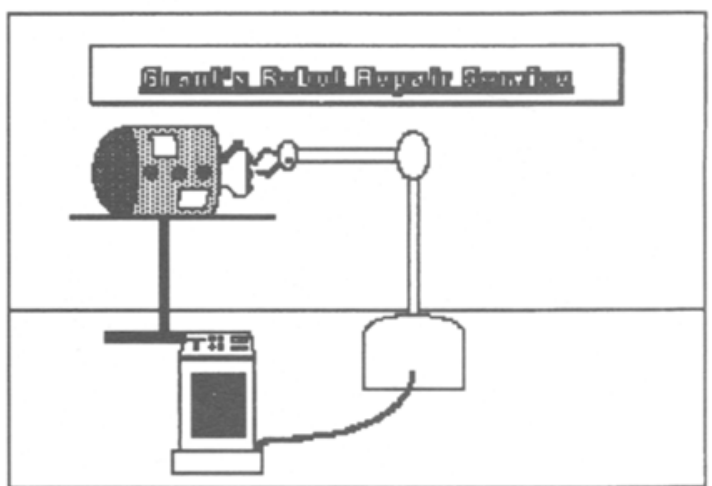

(C)

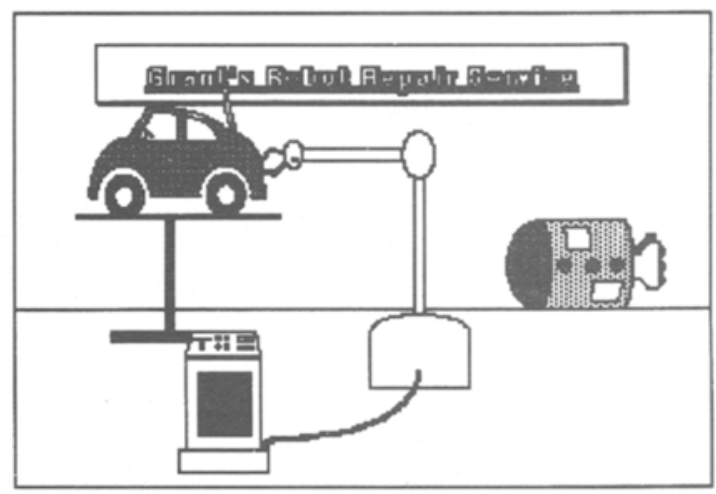

(E)

Figure 5. Sample set of pictures used in Experiment 2.

alignable differences. That is, when subjects rate the similarity of all four pairings of standard with alternative, there should be a greater difference in rated similarity between the two alignable difference pairs (e.g., 5B and 5C) than between the two nonalignable difference pairs (e.g., $5 \mathrm{D}$ and $5 \mathrm{E}$ ). This pattern of data would lead to an interaction between the type of difference and the "similarity" of the added item to the corresponding item in the alignable difference.

\section{EXPERIMENT 2}

\section{Method}

Subjects. The subjects in this study were 24 members of the Columbia University community who were paid for their participation.

Design. There were two levels of item similarity (similar/dissimilar) and two levels of difference type (alignable/nonalignable). Both factors were run within subjects. Order of stimulus presentation was determined randomly for each subject with the restriction that the subject never rate two pairs from the same stimulus set in a row. 
Materials. Eight sets of pictures were generated. Each set consisted of a standard and four comparison figures. In each comparison figure, a new local item was added either as an alignable difference or as a nonalignable difference. The new item was similar to the corresponding item in the standard picture in one of the alignable difference alternatives and was dissimilar to the corresponding item in the other. The same two local items were added to form the two nonalignable difference alternatives. Similarity between the local items of the two alignable difference pairs and the standard's items (and thus between corresponding items) was determined by our intuitions, and was later confirmed by the ratings in the similarity task. The stimulus set is summarized in Table 2 .

Stimuli were drawn with a computer graphics package. Each stimulus pair was placed on a sheet of paper. The standard was always on top. At the bottom of the page was a similarity scale ranging from 1 (labeled highly dissimilar) to 9 (labeled highly similar). The $32 \mathrm{stim}$ ulus pairs were organized into booklets. The instructions were placed on the first page of the booklet.

Procedure. The subjects were given the booklet with the stimulus materials and were seated at a table. The subjects were asked to look at the pictures and to rate the similarity of the pair on the scale provided; they were encouraged to look through the booklet prior to making the ratings to get a feel for the range of materials in the study. The rating task took approximately $10 \mathrm{~min}$ to complete.

\section{Results}

The results bear out the prediction that deviations from the standard matter more in computing similarity if they are alignable with elements of the standard than if they are not. The mean rated similarity of the four kinds of picture pairs for each stimulus set is presented in Table 2 . A $2 \times 2$ repeated measures analysis of variance (ANOVA) was performed on the mean rated similarity for each item. There were main effects of both item similarity and difference type. On average, similar items $(M=6.58)$ yielded higher similarity ratings than did dissimilar items $(M=5.67)$ $[F(1,7)=12.96, p<.05]$. Also, as we found in the pilot studies, nonalignable difference pairs $(M=6.64)$ yielded higher similarity ratings than did alignable difference pairs $(M=5.61)[F(1,7)=10.63, p<.05]$. This difference suggests that a given mismatch counts more against similarity as an alignable difference than as a nonalignable difference. As predicted, there was an interaction between item similarity and difference type $[F(1,7)=8.19, p<.05]$. This interaction reflects that, as expected, the rated similarity for similar alignable difference pairs $(M=6.33)$ was significantly greater than the rated similarity for different alignable difference pairs $(M=4.88)[t(7)=3.76, p<.05$,
Bonferroni], while the difference in rated similarity between similar nonalignable difference pairs $(M=6.82)$ and dissimilar nonalignable difference pairs $(M=6.46)$ was not significant $[t(7)=1.61, p>.05]$. The same pattern was obtained in a subject analysis. The difference in rated similarity between similar and dissimilar alignable difference pairs $(M=1.45)$ was significantly larger than the difference in rated similarity for similar and dissimilar nonalignable difference pairs $(M=0.35)[t(23)=5.92$, $p<.001]$.

\section{Discussion}

These findings extend our previous work by providing an explicit demonstration that the impact of alignable differences on rated similarity is greater than the impact of nonalignable differences on similarity. First, as in the pilot studies, alignable difference pairs were given lower similarity ratings than were nonalignable difference pairs. Second, as predicted, subjects were much more sensitive to the degree of difference for alignable differences than for nonalignable differences. This is consistent with the claim that similarity comparisons focus on alignable differences over nonalignable differences. As discussed above, this focus on alignable differences does not imply that any nonalignable difference will be disregarded, nor that any given alignable difference will greatly decrease similarity. In one stimulus set, a robot fixes a car in the standard and a truck in the alignable comparison figure. While these pictures are clearly different, subjects' overall assessment of similarity was still quite high $(M=6.54$ out of 9.00$)$. The point is that variations in this local item similarity (e.g., replacing the truck by a robot) had a much greater impact on overall similarity (thereby lowering the rated similarity to 4.75) than did parallel variations within the nonalignable difference alternatives. Thus, while both alignable and nonalignable differences count against similarity (all pairs were given ratings less than 9 on the scale), the subjects were much more sensitive to variations in the properties appearing as an alignable difference than to variations in the properties appearing as nonalignable differences.

\section{GENERAL DISCUSSION}

The studies presented here support the view that similarity comparisons involve an alignment of structured rep-

Table 2

Summary of Stimulus Set for Experiment 2 and Similarity Ratings for the Four Conditions of This Study

\begin{tabular}{|c|c|c|c|c|c|c|c|}
\hline \multirow[b]{2}{*}{ Relation } & \multirow{2}{*}{$\begin{array}{l}\text { Target } \\
\text { Object }\end{array}$} & \multirow{2}{*}{$\begin{array}{l}\text { Similar } \\
\text { Object }\end{array}$} & \multirow{2}{*}{$\begin{array}{c}\text { Dissimilar } \\
\text { Object }\end{array}$} & \multicolumn{2}{|c|}{ Alignable Difference Pairs } & \multicolumn{2}{|c|}{ Nonalignable Difference Pairs } \\
\hline & & & & Similar & Dissimilar & Similar & Dissimilar \\
\hline Shooting & Target & Bottle & Bird & 5.67 & 4.67 & 7.88 & 7.50 \\
\hline Painting & Large painting & Small painting & Sculpture & 5.38 & 4.83 & 7.17 & 6.17 \\
\hline Reaching & Cookies & Cake & Cereal & 7.38 & 6.38 & 6.75 & 6.13 \\
\hline Giving & Woman & Squirrel & Garbage can & 7.08 & 4.71 & 6.96 & 7.25 \\
\hline Cooking & Hot dog & Marshmallows & Flag & 7.00 & 3.38 & 7.25 & 6.04 \\
\hline Rescuing & Child & Infant & Cat & 6.54 & 5.63 & 6.42 & 7.08 \\
\hline Repairing & Car & Truck & Robot & 6.54 & 4.75 & 6.17 & 5.58 \\
\hline Fighting & Man & Large man & Small man & 5.04 & 4.67 & 5.96 & 5.92 \\
\hline Mean & & & & 6.33 & 4.88 & 6.82 & 6.46 \\
\hline
\end{tabular}


resentations (Falkenhainer et al., 1989; Gentner, 1983, 1989; Gentner \& Markman, 1994, 1995; Goldstone, 1994; Goldstone \& Medin, 1994a; Goldstone, Medin, \& Gentner, 1991; Markman \& Gentner, 1993a, 1993b; Medin et al., 1993). This view assumes that connections are of paramount importance in mental representations, and that these connections are established via labeled and directed relations. This pattern of connections is crucial to the structural alignment process.

Experiment 1 tested whether three prior findings obtained with verbal stimuli (Gentner \& Markman, 1994; Markman \& Gentner, 1993a) would generalize to pictorial stimuli. The results showed that, as predicted, alignable differences were positively correlated with commonalities and nonalignable differences were not. Furthermore, this study demonstrated explicitly that alignable differences arise from corresponding elements in a pair. Finally, these results yielded greater between-subject consistency for listings of alignable differences than for listings of nonalignable differences, as would be expected if comparisons focused subjects on differences connected to the common structure.

Although the results of Experiment 1 strengthened the evidence for the structural alignment account, it still remained to be demonstrated that subjects' subjective assessments of similarity were affected differentially by alignable and nonalignable differences. The results of Experiment 2 showed that the degree to which an alignable difference decreased similarity varied greatly with its similarity to the corresponding item in the standard. In contrast, variations in an item serving as a nonalignable difference did not much affect overall similarity. These findings bear out the prediction that alignable differences are a more focal part of similarity comparisons than are nonalignable differences.

\section{Generality}

These studies show that the relationship between commonalities and alignable differences is not specific to word pairs. In addition, the present results suggest that the distinction between alignable and nonalignable differences is general to the comparison process and not specific to taxonomic categories. Other research suggests that these patterns are not specific to any particular level of taxonomic abstraction either. For example, as we found here, Markman and Wisniewski (1996) demonstrated that subjects asked to list commonalities and differences of pairs made up of subordinate-, superordinate-, and basic-level concepts listed more commonalities and alignable differences for similar pairs than for dissimilar pairs at all levels of abstraction. Interestingly, Markman and Wisniewski found that basic-level categories were the most abstract categories at which many commonalities and alignable differences could be obtained in comparisons, and speculated that the basic-level advantage in categorization might be related to this ability to fluently compare items.

In general, we find that highly similar pairs have many commonalities and many alignable differences. This pattern has been obtained when the highly similar pairs con- sisted of pairs of categories from the same ontological class (Markman \& Gentner, 1993a), pairs of subordinate categories from the same basic-level category (Markman \& Wisniewski, 1996), and pairs of pictures in which similar objects take part in similar actions (in the present studies). Clearly, however, similarity taken to the limit yields identical pairs, which have many commonalities and no differences. Thus, we are left with the paradox that highly similar pairs have many commonalities and many alignable differences, but the most similar pairs (i.e., identical pairs) have many commonalities and no differences. Further research must determine whether the observed numerical relationship between commonalities and alignable differences holds for all highly similar pairs that are not identi$\mathrm{cal}$, or whether at some point prior to identity the positive relationship between commonalities and alignable differences is no longer obtained.

The present data underline the importance of representational connectivity on the determination of differences. However, a robust effect found across several experiments (Clement \& Gentner, 1991; Goldstone, 1994; Goldstone et al., 1991; Markman \& Gentner, 1993b) is that the importance of a feature match on similarity also depends on its relationship to the overall alignment. For example, Clement and Gentner found that subjects asked to judge which of two matching facts contributed most to an analogy chose the one that was connected to a causal antecedent that matched a causal antecedent in the other passage. A similar point was made by Markman and Gentner (1993b) and Rattermann, Gentner, and DeLoache (1990), who found that the objects that people tended to place in correspondence in a pair of pictures were those that played similar roles in a matching relational structure.

\section{Factors That Influence Alignment}

The use of picture pairs in Experiment 1 afforded us the opportunity to look at the factors that lead subjects to form correspondences. Based on the local-to-global algorithm employed by computational models of structural comparison (Falkenhainer, Forbus, \& Gentner, 1986, 1989; Goldstone, 1994; Holyoak \& Thagard, 1989; Keane \& Brayshaw, 1988), we expected to find - and did indeed find - that items were sometimes placed in correspondence on the basis of local attribute similarities, for example, the matching Christmas trees in Figures 2A and 2B. A prediction that is both more specific and more important to the structuremapping approach is that frequently items were placed in correspondence because they played the same role in a matching relational structure. For example, the angel in Figure $2 \mathrm{~B}$ was typically placed in correspondence with the star in Figure 2A on the basis of their like positions atop the matching trees in the pictures. Subjects' willingness to place nonidentical objects in correspondence, if by so doing they could preserve a systematic structural alignment, is consistent with previous findings (e.g., Clement \& Gentner, 1991; Gentner \& Schumacher, 1986; Gentner \& Toupin, 1986; Goldstone \& Medin, 1994a, 1994b; Halford, 1993; Markman \& Gentner, 1993b; Spellman \& Holyoak, 1993). 
This analysis suggests that attribute alignable differences are often the result of relational correspondences in a scene. According to structural alignment, however, the opposite pattern should not be obtained: attribute matches should not lead to the determination of relational alignable differences. This prediction is based on the fact that relational correspondences are used to constrain the set of object matches, but that attribute correspondences are not used to search for relational matches. Consistent with this prediction, only 2 of the 72 consensus alignable differences (i.e., those listed by 5 or more subjects) were relational alignable differences that were related to attribute correspondences.

In sum, structural alignment posits that the overall representational alignment, and not simply a summation of object similarities, determines scene similarity. Object alignment depends not only on the two compared objects' featural commonalities and differences, but also on the best relational alignment between the scenes. In some comparisons (e.g., literal similarity matches) both the attribute and the relational information correspond, making the object correspondences in these pairs very robust.

\section{Structural Alignment in Cognitive Processing}

From a functional perspective, it makes sense that the comparison process should promote large structural alignments. Such a preference for systematicity in interpretation maximizes the chance of perceiving and using important predictive information such as common causal relations and other higher order patterns. Moreover, people can use differences that are connected to common causal systems as the basis for new causally relevant predictions from the base to the target, and these inferences can be used to increase the systematicity (i.e., coherence) of the target (Bowdle \& Gentner, 1996; Clement \& Gentner, 1991; Gentner \& Bowdle, 1994). Another point to consider on the ecological side is that in everyday reasoning situations the comparison items will typically share relevant commonalities. Their alignable differences - those connected to the common system - are thus likely to be those most relevant to current concerns.

We suggest that structural alignment is a general comparison mechanism. We have also suggested that implicit or explicit comparisons are a central part of many other cognitive processes. Given this assumption, alignable differences should be crucial to other cognitive processes beyond direct similarity judgments. For example, Goldstone (1994) found that similarity increased subjects' sensitivity to alignable properties in a perceptual matching task (see also Goldstone \& Medin, 1994a). In this study, subjects saw scenes composed of two pairs of butterflies displayed briefly on the screen, in which there were certain overall alignments, for example, top butterfly on left $\rightarrow$ top butterfly on right, and so forth. After the screen was erased, subjects rated the similarity of the scenes; they were then asked to indicate whether aligned butterflies or unaligned butterflies had matching values along a given dimension (e.g., were the wings shaded in the same way). A signaldetection analysis indicated significantly greater sensitiv- ity $\left(d^{\prime}\right)$ for feature matches and mismatches for aligned butterflies than for nonaligned butterflies. Thus, comparing scenes facilitates the determination of both matching and mismatching features of corresponding objects.

Structural alignment theory also applies fruitfully in decision making. For example, Tversky's (1972) elimination by aspects (EBA) model compares the items in a choice set on the basis of their values on a common dimension. Items with unsatisfactory values on that dimension are eliminated. This process is repeated until one item remains. Thus, EBA focuses on alignable differences of items in the choice set. Markman and Medin (1995) tested the importance of alignable differences in choice directly. They compared subjects' justifications for choices between pairs of video games with commonality and difference listings for the same pairs obtained from a separate group of subjects. In their justifications, subjects consistently used alignable differences rather than nonalignable differences, suggesting that alignable differences played a greater role in choice processing than did nonalignable differences. Likewise, Johnson $(1988,1989)$ found that people were more likely to compare items in a choice set, and to rely on comparable attributes of the choices, when the items were similar than when they were dissimilar.

Some recent evidence on the creative extension of known categories is also consistent with the structural alignment view. Karmiloff-Smith (1990) asked children to draw pictures of people and houses that "do not exist." She found that children often drew new items that were similar to known items but had alignable differences with them. For example, a "person that does not exist" might have three eyes or six legs. Ward $(1994,1995)$ found a similar pattern with adults. He asked college students to draw fantasy animals, and they too tended to have alignable differences with known animals. Furthermore, he notes that new inventions often maintain the structure of prior artifacts with just those alignable differences needed for the new function (Ward, 1995). For example, early trains were built to resemble stagecoaches running on tracks instead of on roads (even to the extent of retaining such maladaptive qualities of the original as having the conductor and the brakeman sitting on the outside of the car).

Structural alignment may also illuminate the wellknown distinctiveness effects in memory (Hunt, 1995; Hunt \& McDaniel, 1993). In the von Restorff effect, an item is well remembered when it is embedded in a list context that makes it distinctive (e.g., a number may be well remembered in a list of nonsense syllables). Hunt and McDaniel (1993) suggest that structural alignment, with its clear connection between commonalities and alignable differences, may provide the basis for an explanation of these phenomena.

Finally, both ease of alignment and the distinction between alignable and nonalignable differences are important in conceptual combination. Two main strategies have been uncovered for the way subjects interpret novel noun-noun phrases: slot filling (Murphy, 1988) and property mapping (Wisniewski \& Gentner, 1991). In slot filling, a relation is posited between the concepts (e.g., a zebra chair is a chair that zebras sit in). In property map- 
ping, a property of one noun is carried over to the second (e.g., a zebra horse is a horse with stripes). Wisniewski (in press) has found that property-mapping definitions are given most frequently when the concepts denoted by the nouns are similar, whereas slot-filling interpretations are given most frequently when the nouns are not similar. This pattern suggests that property mapping may involve structural alignment. Further evidence is provided by Wisniewski and Markman (1993), who compared definitions of noun pairs to commonality and difference listings of the same word pairs, and found that property-mapping definitions selectively made use of the alignable differences rather than of the nonalignable differences of the words. In contrast, slot-filling definitions did not involve either the alignable or the nonalignable differences of the words. Thus, alignment and mapping may be a natural mediator of conceptual combination for comparable terms (Wisniewski \& Gentner, 1991).

\section{Conclusion}

The present work links similarity to the structuremapping process models of analogy and metaphor, thereby providing a more precise descriptive vocabulary for talking about comparison. This alignment process seems to apply to both perceptual and verbal materials across a range of cognitive domains. As with Tversky's contrast model, which permitted a new level of specificity of prediction by incorporating both commonalities and differences into the account of similarity, the structural alignment view increases the specificity of similarity models by suggesting the tripartite distinction of commonalities, alignable differences, and nonalignable differences. We believe that this heightened precision will bear fruit in the study of comparison as a component of cognitive processing more generally.

\section{REFERENCES}

BAssoK, M. (1990). Transfer of domain-specific problem-solving procedures. Journai of Experimental Psychology: Learning, Memory, \& Cognition, 16, 522-533.

BOWDLE, B. F., \& GENTNER, D. (1996). Informativity and asymmetry. Manuscript in preparation.

Clement, C. A., \& Gentner, D. (1991). Systematicity as a selection constraint in analogical mapping. Cognitive Science, 15, 89-132.

Falkenhainer, B., Forbus, K. D., \& GenTNER, D. (1986). The structuremapping engine. In Proceedings of the Fifth National Conference on Artificial Intelligence (pp. 272-277). Los Altos, CA: Morgan Kaufmann.

FaLKenhainer, B., Forbus, K. D., \& GentNer, D. (1989). The structuremapping engine: Algorithm and examples. Artificial Intelligence, 41(1), 1-63.

GENTNER, D. (1983). Structure-mapping: A theoretical framework for analogy. Cognitive Science, 7, 155-170.

GENTNER, D. (1989). The mechanisms of analogical learning. In S. Vosniadou \& A. Ortony (Eds.), Similarity and analogical reasoning (pp. 199-241). Cambridge: Cambridge University Press.

GENTNER, D., \& BowdLE, B. F. (1994). The coherence imbalance hypothesis: A functional approach to asymmetry in comparison. In The Proceedings of the Sixteenth Annual Conference of the Cognitive Science Society (pp. 351-356). Hillsdale, NJ: Erlbaum.

Gent Ner, D., \& Markman, A. B. (1994). Structural alignment in comparison: No difference without similarity. Psychological Science, $\mathbf{5}$, $152-158$
Gentner, D., \& Markman, A. B. (1995). Similarity is like analogy. In C. Cacciari (Ed.), Similarity (pp. 111-148). Brussels: BROPEL.

GeNTNER, D., \& SCHUMACHER, R. M. (1986). Use of structure mapping theory for complex systems. In Proceedings of the 1986 IEEE International Conference on Systems, Man, and Cybernetics (pp. 252-258). New York: IEEE.

GentNer, D., \& ToupIN, C. (1986). Systematicity and surface similarity in the development of analogy. Cognitive Science, 10, 277-300.

GOLDSTONE, R. L. (1994). Similarity, interactive-activation and mapping. Journal of Experimental Psychology: Learning, Memory, \& Cognition, 20, 3-28.

Goldstone, R. L., \& Medin, D. L. (1994a). Similarity, interactiveactivation and mapping. In K. J. Holyoak \& J. A. Barnden (Eds.), Advances in connectionist and neural computation theory: Vol. 2. Analogical connections (pp. 321-362). Norwood, NJ: Ablex.

Goldstone, R. L., \& Medin, D. L. (1994b). The time course of comparison. Journal of Experimental Psychology: Learning, Memory, \& Cognition, 20, 29-50.

Goldstone, R. L., Medin, D. L., \& Gentner, D. (1991). Relational similarity and the non-independence of features in similarity judgments. Cognitive Psychology, 23, 222-264.

HALFORD, G. S. (1993). Children's understanding: The development of mental models. Hillsdale, NJ: Erlbaum.

Hofstadter, D. R., \& Mitchell, M. (1994). An overview of the Copycat project. In K. J. Holyoak \& J. A. Barnden (Eds.), Advances in connectionist and neural computation theory: Vol. 2. Analogical connections (pp. 31-112). Norwood, NJ: Ablex.

HoLYOAK, K. J., \& KоH, K. (1987). Surface and structural similarity in analogical transfer. Memory \& Cognition, 15, 332-340.

HolyoaK, K. J., \& ThaGard, P. (1989). Analogical mapping by constraint satisfaction. Cognitive Science, 13, 295-355.

HuNT, R. R. (1995). The subtlety of distinctiveness: What von Restorff really did. Psychonomic Bulletin \& Review, 2, 105-112.

HUNT, R. R., \& MCDANIEL, M. A. (1993). The enigma of organization and distinctiveness. Journal of Memory \& Language, 32, 421-445.

JoHnson, M. D. (1988). Comparability and hierarchical processing in multialternative choice. Journal of Consumer Research, 15, 303-314.

JoHnson, M. D. (1989). The differential processing of product category and noncomparable choice alternatives. Journal of Consumer Research, 16, 300-309.

KARMILOFF-SMITH, A. (1990). Constraints on representational change: Evidence from children's drawing. Cognition, 34, 57-83.

KeANE, M. T. (1988). Analogical mechanisms. Artificial Intelligence Review, 2, 19-23.

Keane, M. T., \& Brayshaw, M. (1988). The incremental analogy machine: A computational model of analogy. In D. Sleeman (Ed.), Third European working session on machine learning (pp. 53-62). San Mateo, CA: Morgan Kaufmann.

KoloDNER, J. L. (1993). Case-based reasoning. San Mateo, CA: Morgan Kaufmann.

KRUMHANSL, C. L. (1978). Concerning the applicability of geometric models to similarity data: The interrelationship between similarity and spatial density. Psychological Review, 85, 445-463.

Markman, A. B., \& Gentner, D. (1993a). Splitting the differences: A structural alignment view of similarity. Journal of Memory \& Language, 32, 517-535.

Markman, A. B., \& GentNer, D. (1993b). Structural alignment during similarity comparisons. Cognitive Psychology, 25, 431-467.

Markman, A. B., \& Medin, D. L. (1995). Similarity and alignment in choice. Organizational Behavior \& Human Decision Processes, 63, $117-130$

Markman, A. B., \& Wisniewski, E. J.(1996). Similar and different: The differentiation of basic level categories. Manuscript in preparation.

Medin, D. L., Goldstone, R. L., \& Gentner, D. (1993). Respects for similarity. Psychological Review, 100, 254-278.

Murphy, G. L. (1988). Comprehending complex concepts. Cognitive Science, 12, 529-562.

Novick, L. R. (1988). Analogical transfer, problem similarity, and expertise. Journal of Experimental Psychology: Learning, Memory, \& Cognition, 14, 510-520.

Novick, L. R. (1990). Representational transfer in problem solving. Psychological Science, 1, 128-132. 
Rattermann, M. J., Gentner, D., \& Deloache, J. S. (1990). The effects of familiar labels on young children's performance in an analogical mapping task. In The Proceedings of the Twelfth Annual Conference of the Cognitive Science Society (pp. 22-29). Hillsdale, NJ: Erlbaum.

Ross, B. H. (1989). Distinguishing types of superficial similarities: Different effects on the access and use of earlier examples. Journal of Experimental Psychology: Learning, Memory, \& Cognition, 15, 456-468.

SJöBERG, L. (1972). A cognitive theory of similarity. Göteborg Psychological Reports, 2 (10).

Smith, E. E., \& Medin, D. L. (1981). Categories and concepts. Cambridge, MA: Harvard University Press.

SMITH, E. E., \& OSHERSON, D. N. (1989). Similarity and decision making. In S. Vosniadou \& A. Ortony (Eds.), Similarity and analogical reasoning (pp. 60-75). New York: Cambridge University Press

SPEllman, B. A., \& Holyoak, K. J. (1993). An inhibitory mechanism for goal-directed analogical mapping. In Proceedings of the Fifteenth Annual Conference of the Cognitive Science Society (pp. 947-952). Hillsdale, NJ: Erlbaum.

TVERSKY, A. (1972). Elimination by aspects: A theory of choice. $P_{s y-}$ chological Review, 79, 281-299.

TVERSKY, A. (1977). Features of similarity. Psychological Review, 84, 327-352.

WARD, T. B. (1994). Structured imagination: The role of category structure in exemplar generation. Cognitive Psychology, 27, 1-40.

WARD, T. B. (1995). What's old about new ideas? In S. M. Smith, T. B. Ward, \& R. A. Finke (Eds.), The creative cognition approach. Cambridge, MA: MIT Press.

WISNIEWSKI, E. J. (in press). Similarity, slot-filling and propertymapping in conceptual combination. Journal of Memory \& Language.

WISNIEWSKI, E. J., \& GENTNER, D. (1991), On the combinatorial semantics of noun pairs: Minor and major adjustments to meaning. In G. B. Simpson (Eds.), Understanding word and sentence (pp. 241-284). Amsterdam: Elsevier

WisniEWSKI, E. J., \& MARKMAN, A. B. (1993). The role of structural alignment in conceptual combination. In Proceedings of the Fifteenth Annual Conference of the Cognitive Science Society (pp. 1083-1086). Hillsdale, NJ: Erlbaum.

\section{NOTES}

1. Attributes and relations are predicates with truth values. Functions differ from predicates in that they map from a set of arguments onto values other than truth values. For example, a function like color (ball) $=$ red may be used to represent the dimension of color. The same assertion could be represented using color as an attribute, as in red (ball)--or using color as a relation, as in color (ball, red). We assume that how a property is represented will affect the way it is processed. The representations presented in this paper are meant as plausible psychological construals.

2. Although subjects in this study performed both the commonality and difference listing task and the similarity rating task, subjects in our previous studies (Markman \& Gentner, 1993a) performed only one of the tasks. Since the relationship between rated similarity and listed commonalities, alignable differences, and nonalignable differences parallels that in previous studies in which the tasks were not performed together, it is unlikely that the present results are an artifact of having the same subjects perform both tasks.

3. The high correlation between commonalities and alignable differences once again makes multiple regression analysis inappropriate for examining the relationship between rated similarity and listed commonalities, alignable differences, and nonalignable differences. For comparison with previous work, we point out that if this analysis is carried out, a significant positive regression coefficient for commonalities is obtained and nonsignificant coefficients for both alignable and nonalignable differences are found. This lends weight to our suspicion that our previously obtained significant negative coefficient was artifactual. That is, rather than nonalignable differences' leading to low similarity, the causality may be that low-similarity pairs (because they afford few alignable differences) promote a strategy of searching for nonalignable differences. The present use of picture pairs made nonalignable differences easily available for both high- and low-similarity pairs.

4. This analysis requires that the added nonalignable difference does not lead the subject to reinterpret the main action of the picture to form new alignable differences with the other scene.

5. We thank Brian Ross for suggesting this experiment.
(Manuscript received December 30, 1994; revision accepted for publication April 1, 1995.) 